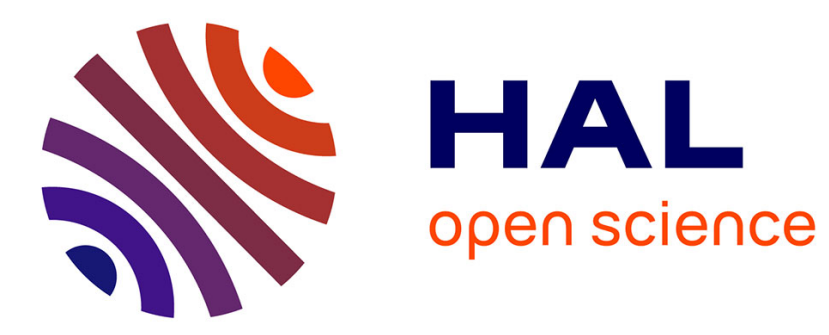

\title{
Cellules souches cancéreuses et cancer du côlon
}

Julie Pannequin

\section{To cite this version:}

Julie Pannequin. Cellules souches cancéreuses et cancer du côlon. Bulletin du Cancer, 2017, 104 (12), pp.1072-1074. 10.1016/j.bulcan.2017.10.016 . hal-02078811

\section{HAL Id: hal-02078811 \\ https://hal.umontpellier.fr/hal-02078811}

Submitted on 5 Jan 2021

HAL is a multi-disciplinary open access archive for the deposit and dissemination of scientific research documents, whether they are published or not. The documents may come from teaching and research institutions in France or abroad, or from public or private research centers.
L'archive ouverte pluridisciplinaire HAL, est destinée au dépôt et à la diffusion de documents scientifiques de niveau recherche, publiés ou non, émanant des établissements d'enseignement et de recherche français ou étrangers, des laboratoires publics ou privés. 


\title{
Cellules souches cancéreuses et cancer du côlon
}

\section{Cancer stem cells in colon cancer}

\author{
Julie Pannequin \\ IGF, Univ. Montpellier, CNRS, INSERM, Montpellier, France
}

Le cancer du côlon est le $4^{\text {ème }}$ cancer le plus meurtrier à travers le monde et son incidence croit dans de nombreux pays probablement à cause du mode de vie occidental qui est de plus en plus adopté et en raison d'un diagnostic souvent trop tardif. En effet, alors que les stades précoces sont associés à une survie de 70 à $90 \%$ à 5 ans, les stades avancés/métastatiques présentent un taux de survie de l'ordre de $10 \%$. Par ailleurs, le taux de récidives n'est pas rare puisqu'environ un patient sur deux développera une nouvelle tumeur dans les 5 ans qui suivent sa prise en charge. Le mauvais pronostic des cancers colorectaux métastatiques et le taux de rechute important sont notamment attribués à la présence des cellules souches cancéreuses qui sont particulièrement résistantes aux thérapies conventionnelles (chimiothérapies et thérapies ciblées dirigées contre le récepteur de l'EGF et contre le VEGF) et qui ont des propriétés particulièrement invasives.

Une des questions les plus récurrentes concernant les CSC est celle de la cellule à l'origine de la tumeur et l'équipe de Hans Clevers, pionnier dans le domaine des cellules souches intestinales, a permis une avancée considérable grâce à l'utilisation de modèles murins transgéniques. En effet, ils ont élégamment montré que la délétion du gène APC, le gène qui lorsqu'il est muté déclenche le processus de tumorigénèse intestinale, précisément dans les cellules souches intestinales, et dans ces cellules uniquement, induisait la formation d'un adénome qui se développera ensuite en carcinome (1). Cette découverte primordiale n’a été 
possible que par l'identification préalable d'un marqueur spécifique des cellules souches intestinales, LGR5 (2). Une avancée majeure a par ailleurs été publiée en 2017 sur la plasticité des cellules souches intestinales exprimant ce même marqueur, LGR5 (3). En utilisant des modèles murins transgéniques, ils ont montré que la déplétion de ces cellules dans la tumeur primaire n'était pas irréversible car d'autres cellules pouvaient se dédifférencier pour pallier à cette disparition. En revanche, si la même déplétion est réalisée dans des métastases hépatiques aucune dédifférenciation n'est possible certainement à cause d'un microenvironnement, une niche, totalement différent. Cette étude pourrait laisser entrevoir des espoirs thérapeutiques en ciblant spécifiquement ces cellules dans les organes distants comme le foie et les poumons.

Comme pour la plupart des cancers, aucun marqueur idéal ne permet de purifier les CSC mais un certain nombre d'entre eux ont été décrits, une liste non exhaustive est présentée dans le paragraphe qui suit.

Les marqueurs des CSC dans le CRC:

CD133 : Alors que ce marqueur est maintenant très controversé, il a été utilisé pour la première identification des CSC dans le CRC par deux équipes en parallèle dans la même issue de Nature en 2007. Ils ont notamment montré par diverses approches in vitro et in vivo que la population de cellules tumorales qui exprimait le marqueur CD133 était beaucoup plus tumorigène que celle qui ne l'exprimait pas $(4,5)$.

- CD44/EpCam ${ }^{\text {High } / C D 166 ~: ~ P l u s ~ t a r d, ~ u n e ~ a u t r e ~ e ́ q u i p e ~ a ~ p r o p o s e ́ ~ c e t t e ~ c o m b i n a i s o n ~ d e ~ m a r q u e u r s ~ s u r ~ l a ~}$ base de ce qui avait été démontré dans le sein (CD44 et EpCam ${ }^{\text {High }}$ ) et CD166 était quant à lui décrit précédemment comme un marqueur de cellules souches mésenchymateuses (6).

CD24: A l'inverse du cancer du sein, l'expression de CD24 est associée avec un phénotype de CSC dans le CRC même si là encore il existe des résultats contradictoires $(7,8)$ 
- CD26 : Cette protéine transmembranaire présentant une activité de protéase a été identifiée comme une cible potentielle d'abord dans le diabète de type 2. Puis en 2010 , une publication importante a démontré que CD26 était un marqueur de cellules souches avec un potentiel métastatique et une chimiorésistance accrue (9). Depuis lors, malheureusement très peu de travaux ont fait l'état de découvertes importantes concernant ce marqueur à l'exception de quelques corrélations entre l'expression de CD26 et une potentielle récidive tumorale chez les patients.

- CD44V6 : Alors que CD44, au même titre que CD133, a été très controversé comme marqueur des CSC dans le CRC, l'isoforme V6 de cette protéine semble très prometteuse. En effet, en 2014 une des équipes pionnières dans le domaine a non seulement démontré que l'expression de CD44V6 marquait une sous population de CSC avec un fort potentiel métastatique mais ils ont également décrypté sa fonction et notamment son association avec c-met et sa liaison avec HGF, 2 facteurs clé de l'invasion tumorale(10).

- Activité aldéhyde deshydrogénase : Cette famille d'enzyme permet de catalyser l'oxydation des fonctions aldéhyde et participe activement à la détoxification des CSC. C'est le premier marqueur fonctionnel décrit pour les CSC dans de nombreux cancers y compris le colorectal (10).

Une des caractéristiques importantes des cellules souches cancéreuses, qu'il ne faut pas négliger au regard de l'explosion de l'immunothérapie notamment dans le contexte du cancer colorectal, est la très faible immunogénicité et l'activité immunosuppressive importante de ces cellules. Certains ont même suggéré que les patients non répondeurs aux traitements PD1/PDL-1 auraient des tumeurs contenant un pourcentage anormalement élevé de CSC. Ce domaine en pleine expansion a définitivement besoin d'éclaircissements notamment pour une meilleure stratification des patients qui pourraient bénéficier de ces traitements.

Un des défis majeurs de ces prochaines années sera le ciblage spécifique des cellules souches cancéreuses colorectales en épargnant les cellules souches intestinales normales. De nombreuses stratégies ont été proposées afin notamment de cibler les CSC en utilisant certains des marqueurs décrits précédemment. Notamment des équipes ont démontré, in vitro mais aussi sur des modèles 
murins précliniques, que l'inhibition de CD133, CD44 ou ALDH1 par différentes approches l'expression diminuaient la tumorigénicité des CSC dans le cancer du côlon (11-13). Aucune de ces stratégies n’a malheureusement débouché sur des essais cliniques très certainement pour un manque de spécificité à la fois des cibles mais également des outils. Une autre manière de faire serait de cibler des propriétés spécifiques des CSC et cruciales pour leur survie. C'est ce qui a été démontré en ciblant spécifiquement Bmi-1, un facteur de transcription qui joue un rôle clé dans l'autorenouvellement. Ce ciblage a un impact fort sur l'initiation et la croissance tumorales in vivo (14). Enfin, le blocage des voies de signalisation particulièrement activées dans les CSC demeure une des plus prometteuses. Les premiers candidats ont été des inhibiteurs des voies Wnt, Notch et Hedgehog et certains d'entre eux sont entrés dans différentes phases précoces d'essais cliniques (RG-4733, Wnt974 et GDC-0449 par exemple). Une stratégie développée récemment semble encourageante, c'est l'inhibition de la voie Stat3 en aval de la kinase Junk (15). Dans cette étude, ils décrivent un inhibiteur de stat3, BBI608, capable d'inhiber le développement métastatique et la récidive tumorale. Cette molécule développée par la compagnie Boston Biomedical est en essai clinique de phase III pour de nombreuses tumeurs solides y compris le cancer colorectal.

Malheureusement toutes les stratégies présentées ci-dessus s'exposent au problème de cibler également les cellules souches intestinales normales et de déclencher potentiellement de sévères effets secondaires. Ainsi, afin de concevoir des stratégies thérapeutiques visant les CSC dans le CRC, le challenge pour les années à venir va être de découvrir des cibles exprimées par les CSC mais absentes ou très peu exprimées dans les cellules souches intestinales normales. C'est le cas par exemple de la progastrine, un précurseur hormonal secrété uniquement par les cellules cancéreuses du côlon et considéré comme un co-carcinogène pour lequel il a été démontré récemment un rôle clé dans la survie des CSC (16). Un blocage de ce précurseur par un anticorps neutralisant pourrait s'avérer une thérapie de différenciation prometteuse. Enfin, la sensibilisation des CSC à la chimiothérapie pourrait également être une bonne piste. C'est ce que propose l'étude de Planque et ses collaborateurs en inhibant l'activité de PXR, un récepteur nucléaire principalement impliqué dans la détoxification des 
hépatocytes en réponse aux xénobiotiques et dont l'expression est négligeable dans les cellules souches intestinales normales (17).

En conclusion, maintenant que le « concept » des CSC dans le CRC est admis au sein de la communauté scientifique, des efforts sont à fournir pour les isoler, les caractériser afin dans un second temps de les cibler le plus spécifiquement possible. 


\section{REFERENCES}

1. Barker N, Ridgway RA, van Es JH, van de Wetering M, Begthel H, van den Born M, et al. Crypt stem cells as the cells-of-origin of intestinal cancer. Nature. 29 janv 2009;457(7229):608-11.

2. Barker N, van Es JH, Kuipers J, Kujala P, van den Born M, Cozijnsen M, et al. Identification of stem cells in small intestine and colon by marker gene Lgr5. Nature. 25 oct 2007;449(7165):1003-7.

3. de Sousa e Melo F, Kurtova AV, Harnoss JM, Kljavin N, Hoeck JD, Hung J, et al. A distinct role for Lgr5(+) stem cells in primary and metastatic colon cancer. Nature. 29 2017;543(7647):676-80.

4. O'Brien CA, Pollett A, Gallinger S, Dick JE. A human colon cancer cell capable of initiating tumour growth in immunodeficient mice. Nature. 4 janv 2007;445(7123):106-10.

5. Ricci-Vitiani L, Lombardi DG, Pilozzi E, Biffoni M, Todaro M, Peschle $\mathrm{C}$, et al. Identification and expansion of human colon-cancer-initiating cells. Nature. 4 janv 2007;445(7123):111-5.

6. Dalerba P, Dylla SJ, Park I-K, Liu R, Wang X, Cho RW, et al. Phenotypic characterization of human colorectal cancer stem cells. Proc Natl Acad Sci U S A. 12 juin 2007;104(24):10158-63.

7. Weichert W, Denkert C, Burkhardt M, Gansukh T, Bellach J, Altevogt P, et al. Cytoplasmic CD24 expression in colorectal cancer independently correlates with shortened patient survival. Clin Cancer Res Off J Am Assoc Cancer Res. 15 sept 2005;11(18):6574-81.

8. Choi D, Lee H-W, Hur K-Y, Kim J-J, Park G-S, Jang S-H, et al. Cancer stem cell markers CD133 and CD24 correlate with invasiveness and differentiation in colorectal adenocarcinoma. World J Gastroenterol. 14 mai 2009;15(18):2258-64.

9. Pang R, Law WL, Chu ACY, Poon JT, Lam CSC, Chow AKM, et al. A subpopulation of CD26+ cancer stem cells with metastatic capacity in human colorectal cancer. Cell Stem Cell. 4 juin 2010;6(6):603-15.

10. Huang EH, Hynes MJ, Zhang T, Ginestier C, Dontu G, Appelman H, et al. Aldehyde dehydrogenase 1 is a marker for normal and malignant human colonic stem cells (SC) and tracks SC overpopulation during colon tumorigenesis. Cancer Res. 15 avr 2009;69(8):3382-9.

11. Elsaba TMA, Martinez-Pomares L, Robins AR, Crook S, Seth R, Jackson D, et al. The stem cell marker CD133 associates with enhanced colony formation and cell motility in colorectal cancer. PloS One. 19 mai 2010;5(5):e10714.

12. Du L, Wang H, He L, Zhang J, Ni B, Wang X, et al. CD44 is of functional importance for colorectal cancer stem cells. Clin Cancer Res Off J Am Assoc Cancer Res. 1 nov 2008;14(21):6751-60.

13. Golubovskaya V, O'Brien S, Ho B, Heffler M, Conroy J, Hu Q, et al. Down-regulation of ALDH1A3, CD44 or MDR1 sensitizes resistant cancer cells to FAK autophosphorylation inhibitor Y15. J Cancer Res Clin Oncol. sept 2015;141(9):1613-31.

14. Kreso A, van Galen P, Pedley NM, Lima-Fernandes E, Frelin C, Davis T, et al. Self-renewal as a therapeutic target in human colorectal cancer. Nat Med. janv 2014;20(1):29-36. 
15. Li Y, Rogoff HA, Keates S, Gao Y, Murikipudi S, Mikule K, et al. Suppression of cancer relapse and metastasis by inhibiting cancer stemness. Proc Natl Acad Sci U S A. 10 févr 2015;112(6):1839-44.

16. Giraud J, Failla LM, Pascussi J-M, Lagerqvist EL, Ollier J, Finetti P, et al. Autocrine Secretion of Progastrin Promotes the Survival and Self-Renewal of Colon Cancer Stem-like Cells. Cancer Res. 15 juin 2016;76(12):3618-28.

17. Planque C, Rajabi F, Grillet F, Finetti P, Bertucci F, Gironella M, et al. Pregnane X-receptor promotes stem cell-mediated colon cancer relapse. Oncotarget. 30 août 2016;7(35):56558-73. 